\title{
Analysis of independent cohorts of outbred CFW mice reveals novel loci for behavioral and physiological traits and identifies factors determining reproducibility
}

\author{
Jennifer Zou, ${ }_{1}^{1}$ Shyam Gopalakrishnan, ${ }^{2}$ Clarissa C. Parker, ${ }^{3}$ Jerome Nicod, ${ }^{4}$ Richard Mott (D) ${ }^{5} \mathrm{Na}$ Cai (D) ${ }^{6}$ Arimantas Lionikas (iD) ? \\ Robert W. Davies, ${ }^{8}$ Abraham A. Palmer (D),${ }^{9,10, *}$ and Jonathan Flint (D) ${ }^{11, *}$ \\ ${ }^{1}$ Department of Computer Science, University of California, Los Angeles, CA 90024, USA, \\ ${ }^{2}$ Faculty of Health and Medical Sciences, GLOBE Institute, University of Copenhagen, Copenhagen DK-1353, Denmark, \\ ${ }^{3}$ Department of Psychology and Program in Neuroscience, Middlebury College, Middlebury, VT 05753, USA, \\ ${ }^{4}$ The Francis Crick Institute, London NW1 1AT, UK, \\ ${ }^{5}$ UCL Department of Genetics, Evolution \& Environment, UCL Genetics Institute, London WC1E 6BT, UK, \\ ${ }^{6}$ Helmholtz Zentrum Muenchen, Helmoltz Pioneer Campus, Neuherberg 85764, Germany, \\ ${ }^{7}$ School of Medicine, Medical Sciences and Nutrition, College of Life Sciences and Medicine, University of Aberdeen, Aberdeen AB24 3FX, UK, \\ ${ }^{8}$ Department of Statistics, University of Oxford, Oxford OX1 2JD, UK, \\ ${ }^{9}$ Department of Psychiatry, University of California San Diego, La Jolla, CA 92093, USA, \\ ${ }^{10}$ Institute for Genomic Medicine, University of California San Diego, La Jolla, CA 92093, USA and \\ ${ }^{11}$ Department of Biobehavioral Sciences, University of California, Los Angeles, CA 90024, USA \\ *Corresponding author: Email: jflint@mednet.ucla.edu (J.F.); aap@ucsd.edu (A.A.P.)
}

\begin{abstract}
Combining samples for genetic association is standard practice in human genetic analysis of complex traits, but is rarely undertaken in rodent genetics. Here, using 23 phenotypes and genotypes from two independent laboratories, we obtained a sample size of 3076 commercially available outbred mice and identified 70 loci, more than double the number of loci identified in the component studies. Fine-mapping in the combined sample reduced the number of likely causal variants, with a median reduction in set size of $51 \%$, and indicated novel gene associations, including Pnpo, Ttll6, and GM11545 with bone mineral density, and Psmb9 with weight. However, replication at a nominal threshold of 0.05 between the two component studies was low, with less than one-third of loci identified in one study replicated in the second. In addition to overestimates in the effect size in the discovery sample (Winner's Curse), we also found that heterogeneity between studies explained the poor replication, but the contribution of these two factors varied among traits. Leveraging these observations, we integrated information about replication rates, study-specific heterogeneity, and Winner's Curse corrected estimates of power to assign variants to one of four confidence levels. Our approach addresses concerns about reproducibility and demonstrates how to obtain robust results from mapping complex traits in any genome-wide association study.
\end{abstract}

Keywords: GWAS; CFW; replication; Winner's Curse; power; mega-analysis

\section{Introduction}

Combining samples, through meta- or mega-analysis, has become routine in human genome-wide association studies (GWAS) of complex traits as a way to augment power by increasing sample size and to ensure robustness of results by replicating findings. Genetic mapping of complex traits in rodents has favored the use of linkage analysis in crosses between inbred strains, with many different inbred strain combinations being employed. In addition, few studies examine the same phenotypes. Therefore, few rodent studies have lent themselves to meta- or mega-analysis (Wuschke et al. 2007; Schmidt et al. 2008). However, the more recent transition to genetic association using outbred mice (Parker and Palmer 2011; Flint and Eskin 2012; Chesler 2014) or panels of inbred animals (Ghazalpour et al. 2012) provides opportunities for deploying meta- (Kang et al. 2014) and mega-analysis (Chitre et al. 2020; Zhou et al. 2020) to increase power and test reproducibility.

In this paper, we combine results from two independent laboratories, one at the University of Oxford (OX) in the United Kingdom (Nicod et al. 2016) and one at the University of Chicago (UC) in the United States of America (Parker et al. 2016). Both experiments sampled from the same population of commercially available outbred mice [Crl:CFW(SW)-US_P08, hereafter CFW], but differed in genotyping platforms, and in some of the phenotyping assays. The combined sample size of 3076 mice is the largest cohort to date for the 23 phenotypes examined. The increased sample size and variety of physiological and behavioral phenotypes provided an opportunity to examine several questions that have not been fully addressed in mouse GWAS. 
While a consensus has arisen in human GWAS that a $5 \times 10^{-8}$ threshold together with replication in an independent sample is sufficient to declare locus discovery (Pe'er et al. 2008; Visscher et al. 2012), the same is not true for mouse studies. Mouse populations used for GWAS differ substantially, in linkage disequilibrium (LD) structure, allele frequencies, and the extent of relatedness between subjects, making it inappropriate to codify a single significance threshold (Flint and Eskin 2012). Furthermore, the selection of a genome-wide significance threshold introduces a bias known as "Winner's Curse" or the "Beavis effect," in which loci passing the genome-wide significance threshold tend to have inflated effect sizes (Zhong and Prentice 2008; Xiao and Boehnke 2009; Sun et al. 2011). Winner's Curse contributes substantially to lack of replication of GWAS loci in follow-up studies (Palmer and Pe'er 2017). There have also been concerns about the impact of laboratory differences on the measurement of behavior (Crabbe et al. 1999) and the potentially large impact of subtle laboratory effects (Valdar et al. 2006; Zhou et al. 2020). These differences between studies can contribute to study-specific heterogeneity, which can lead to spurious associations when combining studies. We refer to unintended effects present in one study but not others as confounders. These issues question the generalizability of genetic analyses.

Here we performed a mega-analysis between the OX and UC cohorts and identified 70 independent loci, of which 41 were not found in component studies. Novel loci can result from the increase in power from combining data in a mega-analysis, or they can result from study-specific heterogeneity between the component studies. To investigate the robustness of the novel associations in the mega-analysis, we integrate assessments of heritability, genetic correlation, locus-specific replication, estimates of study-specific heterogeneity due to confounding (Zou et al. 2020), and Winner's Curse corrected estimates of power (Zhong and Prentice 2008) to categorize loci into one of four confidence levels. We then performed fine-mapping analysis and annotation of nonsynonymous variants to identify putative genes for a number of phenotypes.

\section{Methods Subjects}

All animals originated from the same colony of outbred mice, the Crl:CFW(SW)-US_P08 stock, maintained by Charles River Laboratories in Portage, Michigan at the time of the study. Details about the OX and UC mice are described elsewhere (Nicod et al. 2016; Parker et al. 2016). The OX mice were purchased between December 2009 and September 2011 at 4-7 weeks of age and phenotyping started at 16 weeks. The UC mice arrived at 7 weeks of age between August 2011 and December 2012, and testing started after a 2-week acclimatization period. Both the OX and UC mice were maintained on a standard 12:12 h light-dark cycle with water and standard laboratory chow available ad libitum and housed 3 (OX) or 4 (UC) per cage.

\section{Phenotypes}

The effect of covariates, including sex, weight, and batch, was tested in each data set separately (OX and UC) and introduced in a linear model to calculate residuals when the effect was significant (weight was retained for mapping body weight). OX and UC residuals were then quantile-normalized before being merged in a single data set used for the genetic analysis. Supplementary Data S1 lists the mean and standard deviation of all measures obtained in both studies and the covariates included in the linear model to calculate the residuals. Many of the behavioral phenotypes were not independent. For example, there were multiple phenotypes within the category of locomotor activity. They represent measures related to locomotor activity at different timepoints during the testing period (e.g., 0-15, 15-30 min, etc.) as well as summary data representing changes in activity over time (e.g., activity decay) that are likely correlated. Descriptions of all phenotypes can be found in Supplementary Table S1. The following section describes methodological differences between OX and UC. Further details of methods applied in the individual studies can be found in separate publications (Nicod et al. 2016; Parker et al. 2016).

\section{Locomotor activity}

In both centers the baseline activity of the mice was recorded during the first $30 \mathrm{~min}$ of testing. The activity of the OX mice was recorded at 16 weeks after placement into a new plastic home cage $(46 \mathrm{~cm} \times 15 \mathrm{~cm} \times 21 \mathrm{~cm})$ using a photoactivity system from San Diego Instruments (San Diego, CA, USA) which has seven infrared photobeams crossing the width of the cage floor.

The UC mice, $\sim 51$ days of age at the time of test ( $S D=4$ ), were injected i.p. with physiological saline $(0.01 \mathrm{ml} / \mathrm{g}$ body weight $) \mathrm{im}$ mediately prior to the test and placed in the center of an OF chamber (AccuScan Instruments, Columbus, $\mathrm{OH}$ ) consisting of a clear acrylic arena $(40 \mathrm{~cm} \times 40 \mathrm{~cm} \times 30 \mathrm{~cm})$ placed inside a frame containing evenly spaced infrared photobeams.

Four phenotypes were analyzed for this study: locomotor activity initial (0-15 min), locomotor activity end (15-30 min), locomotor activity total (0-30 min), and activity decay. Activity decay was defined as the decrease in activity from beginning to end.

\section{Conditioned fear}

In OX, conditioned fear (CF) was tested over 2 days in four San Diego Instruments chambers with mice at 17 weeks of age. On the first day of the test mice were subjected to a 13-min training session during which they received two electric foot shocks $(0.3 \mathrm{~mA}, 0.5 \mathrm{~s})$ preceded by a 30 -s tone. In the morning of the second day of the test the mice were placed in the same enclosure for $5 \mathrm{~min}$ and fear associated with the context was measured by the amount of freezing. In the afternoon the animals were placed in a different enclosure for 5 min where they were subjected to two 30-s tones without any paired electric shock. Freezing behavior during all sessions of the test was scored using a VideoTrack automated system (Viewpoint, Champagne Au Mont D'Or, France).

UC mice were tested for CF at $\sim 63$ days of age (SD $=2.9$ ) over three consecutive days, each consisting of a 7-min trial: on the first test day, mice were conditioned to associate a test chamber and a tone $(85 \mathrm{~dB}, 3 \mathrm{kHz}$ tone lasting $30 \mathrm{~s})$ with a shock (2-s, 0.5$\mathrm{mA}$ foot shock delivered four times); on the second test day, the mice were re-exposed to the same context, but no tones or shocks were given; on the third day, mice were exposed to the conditioned stimulus (the tones), but in a different environment. Mice were tested in four chambers obtained from Med Associates (St. Albans, VT, USA) and immobility, or "freezing" behavior, was recorded by analyzing digital video with Freeze Frame software (Actimetrics, Evanston, IL, USA).

Phenotypes from the conditioned fear tests consisted of eight measurements of immobility collected in OX and UC mice. On day 1, we measured average proportion of freezing during the pretraining interval (UC: 30-180 s, OX: 180-355 s) before exposure to tones and shocks (baseline freezing D1), as well as the average proportion of freezing during exposure to the first conditioned 
stimulus corrected for baseline freezing (corrected freezing to tone alone). On day 2, we measured freezing during the context test corrected for baseline (corrected freezing to context). On day 3 , we measured the average proportion of time freezing in the altered setting during the 30-s intervals in which the tones were presented corrected for baseline (corrected freezing to cue). These measurements were done on day 2 in Oxford and on day 3 in UC.

\section{Prepulse inhibition}

UC mice were tested at a mean age of 74.5 days $(S D=2.2)$. During prepulse inhibition (PPI), the mice were exposed to loud pulses $(120 \mathrm{~dB})$ that caused them to exhibit the startle response. Occasionally, the exposure to the loud pulse was preceded by a barely perceptible "prepulse" (3-12 dB over background levels), which inhibited the startle response to varying extents. The UC PPI testing procedures follow protocols detailed in previous papers (Palmer et al. 2000, 2004; Palmer and Airey 2003; Shanahan et al. 2009).

OX mice were tested at $\sim 17$ weeks of age following a different protocol (Yee et al. 2005) combining exposure to loud pulses of three different intensities $(100,110$, and $120 \mathrm{~dB})$, also preceded by 3-12 dB prepulses. For this analysis, only measures of startle elicited by the $120 \mathrm{~dB}$ pulse (similar to UC) were considered.

Both OX and UC prepulse inhibition tests were performed in chambers and apparatus from the same manufacturer and model (San Diego Instruments, San Diego, CA, USA), capturing mouse movement using a piezoelectric accelerometer, then converted into digital data and recorded on a computer. Before the start of each test day, the apparatuses were calibrated according to the manufacturer's instructions.

Five phenotypes were analyzed for this study: startle habituation difference, startle habituation ratio, startle, PPI with $+6 \mathrm{db}$ prepulse, and PPI with $+12 \mathrm{db}$ prepulse. In UC, startle habituation difference was defined as the average startle amplitude during the fourth pulse-alone trials subtracted from the average startle amplitude during the first pulse-alone trials. In OX this was calculated by subtracting the last block from the first block for the $120 \mathrm{~dB}$ stimulus. In UC, startle habituation ratio was defined as the average startle amplitude during the first pulse-alone trials divided by the average startle amplitude during the fourth pulsealone trials. In OX, this was calculated by dividing the first block for the $120 \mathrm{~dB}$ stimulus by the last block. Startle was defined as the average startle amplitude to the $120 \mathrm{db}$ pulses during the second and third blocks. PPI with $+6 \mathrm{db}$ prepulse was defined as the average prepulse inhibition during blocks 1 and 2 to the $6 \mathrm{~dB}$ prepulse. PPI with $+12 \mathrm{db}$ prepulse was defined as the average prepulse inhibition during blocks 1 and 2 to the $12 \mathrm{~dB}$ prepulse.

\section{Musculoskeletal traits}

Weight of hind limb muscles and length of tibia were collected by the same experimenter for both the OX and UC mice. Following sacrifice, at $\sim 20$ weeks for the OX mice and $~ 90$ days of age for the UC mice $(M=91.2, S D=2.6)$, one leg was cutoff just below pelvis, tubed, and transferred into a $-70^{\circ} \mathrm{C}$ freezer, then shipped on dry ice to $\mathrm{Dr}$ Arimantas Lionikas at the University of Aberdeen. On the day of dissection, the leg was defrosted and two dorsiflexors, tibialis anterior (TA) and extensor digitorum longus (EDL), and three plantar flexors, gastrocnemius (gastroc), plantaris, and soleus, were removed under a dissection microscope and weighed to a precision of $0.1 \mathrm{mg}$ on a balance (Pioneer, Ohaus). Then, the soft tissues were stripped off from the tibia, and the length of the tibia was measured to a precision of $0.01 \mathrm{~mm}$ with a digital caliper (Z22855, OWIM GmbH \& Co).

\section{Bone mineral}

In the OX mice, bone mineral content of the tibia was measured with the Faxitron MX-20 scanner (Faxitron Bioptics LLC, AZ, USA) using methods adapted from (Bassett et al. 2012). ImageJ (V1.48p, National Institutes of Health, USA) was used to quantify the apparent bone mineral content and the mean of the value obtained for the entire bone used for analysis. In the UC mice, bone mineral density for the entire isolated femur was assessed by Dual X-ray absorptiometry using a GE-Lunar PIXImus II Densitometer (GE-Lunar, Madison, WI, USA). To admit a normal distribution, we transformed the BM measurements, which were ratios, to the (base 10) log-scale. Lastly, we measured abnormal BMD, which is a dichotomized version of the BMD phenotype, and the only phenotype collected that was not quantitative.

\section{Body weight and tail length}

Body weight was measured at $\sim 17$ weeks in OX mice and at $\sim 13$ weeks of age $(91.2, \mathrm{SD}=2.6$ ) in UC mice. Tail length (in $\mathrm{cm}$ ) was measured at the time of sacrifice, as the distance from the base of the tail to the tip of the tail (OX: 20 weeks of age; UC: same as body weight measurement).

\section{Genotypes}

Sample BAM preprocessing including mapping and quality control is as previously described (Nicod et al. 2016; Parker et al. 2016). Reads were aligned to the reference genome assembly (NCBI release 38, mm10). Imputation was carried out using STITCH (Davies et al. 2016) (Supplementary Methods). The 3.15 M SNPs from STITCH were filtered for low quality variant calls (INFO < 0.90) and pruned for LD using PLINK (Purcell et al. 2007), resulting in 97,452 SNPs tested for association with the phenotypes. Using PLINK's hard calling threshold, the dosages were converted to genotype calls. Jointly calling the OX and UC samples in this manner generated a high confidence set of SNPs that was different from those used in their prior studies (Nicod et al. 2016; Parker et al. 2016).

\section{Mapping of traits in the combined set}

The genotype likelihoods at 97,452 independent SNPs, obtained using STITCH, were used to perform associated studies in the joint set of Oxford and Chicago samples. The genotype likelihoods were converted to dosages of the alternate allele. Using the dosages for the combined set of samples, association analyses were performed using linear mixed models in GEMMA (v0.96) (Zhou and Stephens 2012) with a precalculated genetic relationship matrix (GRM) also from GEMMA. Phenotypes were converted to normal quantiles after removing the effects of age and sex, all analyses performed using the $\mathrm{R}$ progamming language (R Development Core Team 2010). We did not convert into normal quantiles within sex because sex was used as a covariate to generate the residuals, thus removing the difference in the means. In addition to performing the association analyses on the combined set of Oxford and Chicago samples, association analyses were also performed on the individual sample sets (OX and UC). Finally, the Oxford sample set was randomly split into two equal sized samples sets 100 times. Association analyses were performed on these Oxford subsets as well. Independent QTLs were obtained using a peak caller (Nicod et al. 2016), where the QTLs with the highest log P-values were retained. The code for 
the peak caller is available on the Github repository (Data availability).

\section{Computation of significance thresholds for association analyses}

The significance thresholds for the P-values from the linear mixed model association analyses were computed in two different permutation approaches. First, we used a "naïve" permutation approach (Cheng and Palmer 2013). In this approach, for each phenotype, we permuted the phenotype values across the combined set of samples. Then the association analysis was repeated on the permuted phenotypes, and the most significant Pvalue was retained. For each phenotype, the naïve permutation analysis was repeated 100 times, resulting in a total of 2300 most significant $P$-values. The permutation threshold was computed as the fifth percentile of the distribution of the most significant Pvalues from across all phenotypes; combining the phenotypes was justified because all phenotypes had been converted to normal quantiles, therefore they had identical distributions. The same permutation threshold was then used for all phenotypes. Separate P-value permutation thresholds were computed for the different sample sets: combined, OX, UC, and the two Oxford subsets (OX1 and OX2).

We compared the naïve permutation method to using another permutation approach-henceforth referred to as the "decorrelated" method (Abney 2015). First, the centered GRM was computed using the genotype dosages from all the chromosomes. Then the inverse square root of the GRM was computed. The phenotype and genotype vectors are premultiplied by the inverse square root of the GRM, resulting in decorrelated measurements. Since the phenotypes and the genotypes are decorrelated, a linear model is used instead of a linear mixed model, resulting in faster computation times. For each phenotype, 100 permutation replicates were performed by permuting the decorrelated phenotypes and performing association analyses using linear models. Similar to the naïve approach, the most-significant P-value was retained from each permutation replicate across the 2300 phenotype-replicate combinations. Again, the significance threshold was computed as the 5th percentile of the distribution of the most-significant P-values. The significance thresholds obtained using the decorrelated method were close to the naive permutation method (Supplementary Figure S6). Since the decorrelated method was less computationally intensive, and the two approaches led to very similar significance thresholds, we use the thresholds obtained using the decorrelated method for our results.

\section{Genetic correlation}

We constructed local LD weighted genetic relatedness matrices (GRMs) using imputed dosages at the common set of SNPs for Oxford and Chicago studies separately, and a combined GRM for all mice in both studies, using LDAK version 5.9 (Speed et al. 2012). We then inferred "narrow-sense" heritability from genome-wide SNPs (h2) at 23 phenotypes measured in both studies, and genetic correlations $(\mathrm{rG})$ between the two component studies in phenotypes with nonzero heritability. Estimates for h2 at each phenotype were obtained using the individual study GRMs using restricted maximum likelihood (REML) implemented in LDAK, while rG for each phenotype was estimated with the combined GRM using bivariate REML implemented in GCTA version 1.93.2 (Yang et al. 2011). All h2 and rG calculations were made with PCs 1 to 20 from PCA on the respective GRMs included as fixed effect covariates. We performed a mega-analysis for all phenotypes with significantly nonzero $r G(P<0.05)$.

\section{Confidence intervals and fine-mapping}

Confidence intervals (CIs) were estimated by simulation. At each QTL, a residual phenotype was constructed by removing the effect of the top SNP at the QTL from the phenotype vector used in the QTL mapping above. This abolished the effect attributable to the QTL whilst maintaining genetic contributions from elsewhere in the genome. One thousand SNPs were then randomly selected, subject to the constraint that they were within $2.5 \mathrm{Mb}$ of the top SNP and were polymorphic in the subset of individuals phenotyped for the trait. A causal variant was simulated at the SNP, with an effect size matching that of the top SNP, taking account of the allele frequency, and added to the residual phenotype. A local scan of the region using the same mixed model but the simulated phenotype was performed and the location and $\log P$ of the simulated top SNP recorded. Across the 1000 simulations, we estimated the distribution of the drop $\Delta$ in $\log P$ between the simulated top SNP and the simulated causal SNP (this was zero when the top and causal SNPs coincided). We used the fraction of simulations $f(\Delta)$ within $\Delta$ to determine CIs for the original phenotype data. Thus, we identified the range of SNPs within $1.5 \mathrm{Mb}$ of the top SNP and with a $\log$ P drop less than $\Delta$ to define the $100 f(\Delta) \%$ CI for the QTL. We used the pruned SNPs within the $95 \%$ CIs as input to the SusieR fine-mapping method. We ran the SusieR method (Wang et al. 2020) using the susie_rss function with $L=1$ and coverage $=0.95$. We annotated all variants within the $95 \%$ causal sets and all variants in perfect LD using ANNOVAR (Wang et al. 2010)

\section{Modeling Winner's Curse and study-specific heterogeneity}

We applied two random effects models proposed in Zou et al. (2020). The $z$-scores of the significant variants in the discovery study and the corresponding $z$-scores in the replication study are used as input to learn the three unknown parameters $\left(\sigma_{g}{ }^{2}, \sigma_{\mathrm{c} 1}{ }^{2}, \sigma_{\mathrm{c} 2}{ }^{2}\right)$ using maximum likelihood. $\sigma_{g}{ }^{2}$ is the variance in the true effect size. $\sigma_{c 1}{ }^{2}$ and $\sigma_{\mathrm{c} 2}{ }^{2}$ are the variances in the study-specific effects for the discovery and replication studies, respectively. The first model (WC) only corrects for Winner's Curse and assumes that the discovery and replication studies have a shared genetic effect $\left(\lambda \sim N\left(0, \sigma_{g}{ }^{2}\right)\right)$. This model corrects for Winner's Curse by modeling the conditional distribution of the replication statistics given discovery statistics $\left(s_{k}^{(2)} \mid s_{k}^{(1)}=x, \sigma_{g}^{2} \sim N\left(\frac{\sqrt{N_{1} N_{2} \sigma_{g}^{2}}}{N_{1} \sigma_{g}^{2}+1} x, 1+N_{2} \sigma_{g}^{2}-\frac{N_{2} \sigma_{g}^{2}}{N_{1} \sigma_{g}^{2}+1}\right)\right)$, where $N_{1}$ and $\mathrm{N}_{2}$ are the sample sizes of the discovery and replication studies, respectively, and $k$ is a genetic locus used as input to the method. The second $(\mathrm{WC}+\mathrm{C})$ corrects for both Winner's Curse and studyspecific effects for the discovery $\left(\delta^{(1)}\right)$ and replication studies $\left(\delta^{(2)}\right)$, where $\delta^{(1)} \sim \mathrm{N}\left(0, \sigma_{\mathrm{c} 1}{ }^{2}\right)$ and $\delta^{(2)} \sim \mathrm{N}\left(0, \sigma_{\mathrm{c} 2}{ }^{2}\right)$. This model corrects for both Winner's Curse and study-specific heterogeneity using a similar conditional distribution $\left(s_{k}^{(2)} \mid s_{k}^{(1)}=x, \sigma_{g}{ }^{2}, \sigma_{c 1}{ }^{2}, \sigma_{c 2}{ }^{2}\right.$ $\sim N\left(\frac{\sqrt{N_{1} N_{2}} \sigma_{g}^{2}}{N_{1} \sigma_{c 1}{ }^{2+} N_{1} \sigma_{g}{ }^{2}+1} x, 1+N_{2} \sigma_{g}^{2}+N_{2} \sigma_{c 2}^{2}-\frac{N_{1} N_{2} \sigma_{g}{ }^{4}}{N_{1} \sigma_{c 1}{ }^{2+} N_{1} \sigma_{g}{ }^{2}+1}\right)$, where $x$ is the value of the $z$-score in the discovery study. We apply this method only to the nine phenotypes with at least three significant loci.

We compared the expected replication rate under the two models to the observed replication. The observed replication was computed as the fraction of variants that were significant in discovery study that were also nominally significant and having the same 
sign in the replication study. The expected replication rate $(r)$ under each model was computed as $r=\frac{1}{M} \sum_{k=1}^{M} P\left(\operatorname{abs}\left(s_{k}{ }^{(2)}\right)>z \mid s_{k}{ }^{(1)}=x\right)$, where $M$ is the number of variants significant in the discovery study, and $\mathrm{z}$ is the significance threshold for the replication study.

\section{Power calculations}

We estimated the power of the association statistics obtained from the OX, UC, and combined data sets using (Zhong and Prentice 2008) to correct for Winner's Curse. This method corrects for the bias from Winner's Curse and provides an estimate of the true effect size $\left(\beta_{\text {true }}\right)$ as follows:

$$
\beta_{\text {true }}=\beta_{\text {obs }}-\sigma \frac{\phi\left(\left(\frac{\beta}{\sigma}\right)-c\right)-\phi\left(\left(-\frac{\beta}{\sigma}\right)-c\right)}{\psi\left(\left(\frac{\beta}{\sigma}\right)-c\right)-\psi\left(\left(-\frac{\beta}{\sigma}\right)-c\right)},
$$

where $\beta_{\text {obs }}$ is the observed effect size, $\sigma$ is the standard error, $\mathrm{c}$ is the significance threshold, $\phi(x)$ is the standard normal density function, and $\psi(x)$ is the standard normal cumulative density function.

We use the estimates of the true effect size to compute the power as the probability of observing a significant result under a normal distribution $\left(s_{k}{ }^{(1)} \sim N\left(\left(\beta_{\text {true }} / \sigma\right), 1\right)\right)$.

\section{Results}

We provide an overview of our methodology in Figure 1, which shows the numbers of phenotypes, QTLs, and genes that we identified at each stage of the analysis.

\section{Combining phenotypes and genotypes}

We identified 23 phenotypes that were measured in both studies (Supplementary Table S1 and Data S1). We observed a high correlation between related phenotypes, such as "locomotor activity initial" and "locomotor activity total" (Supplementary Figure S1). To obtain a common set of genotypes, we converted sequence data into genotypes using a single pipeline and obtained a common set of 3,152,108 SNPs for mapping with MAF $>0.001$. Quality control data for genotypes are shown in Supplementary Figures
S2-S4. We also genotyped a subset of these mice on the megaMUGA array (Collaborative Cross Consortium 2012), and observed $98.71 \%$ concordance for the OX cohort and $97.14 \%$ concordance for the UC cohort. We also compared the overlapping SNPs to our prior publications and found $99.12 \%$ concordance for OX and $91.87 \%$ concordance for UC. After filtering the imputed genotypes for pairwise $r^{2}$ (>0.999), 97,452 SNPs were retained for subsequent mapping (Supplementary Data S2). For all phenotypes, residuals were obtained by regressing out the relevant covariates in a linear model. These residuals were converted to normal quantiles within each cohort and then combined for the megaanalysis.

\section{Genetic correlations}

To determine the degree of concordance between the OX and UC component studies, we computed genetic correlations using bivariate genome-based restricted maximum likelihood estimates, implemented in the GCTA software package (Yang et al. 2011) (Figure 2). Genetic correlations were obtained for 17 of the 23 phenotypes with nonzero heritabilities (Supplementary Table S2) using samples from both studies. We use these 17 phenotypes for further analyses since phenotypes with low heritability and genetic correlation are unlikely to have genome-wide significant associations. For these analyses, we used a set of pruned variants with INFO $>0.90$. As a control, we performed the same analyses using two cohorts that were obtained by randomly splitting the OX cohort in half (termed the OX1 and OX2). Theoretically, there should be little heterogeneity between OX1 and OX2, so the genetic correlation should be close to 1 .

As expected, the genetic correlation between OX1 and OX2 was indeed close to 1 for all phenotypes (Supplementary Figure S5). The standard errors on the genetic correlations were high when the estimated heritability for the trait was low. Figure 2 shows that genetic correlations between OX and UC were $>0.7$ for phenotypes with heritability greater than or equal to $15 \%$, indicating that the studies have substantial shared genetic association signal. Behavioral phenotypes, such as startle and fear conditioning, tended to have lower heritabilities (Supplementary

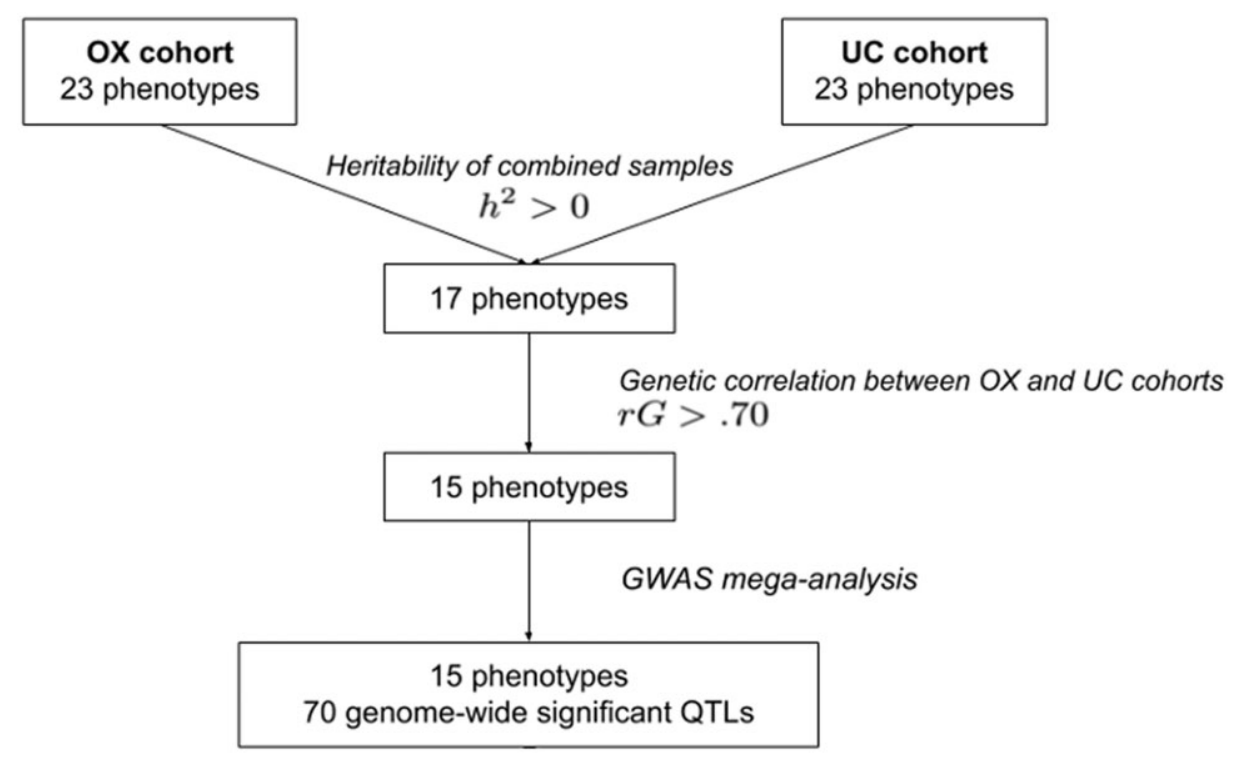

Figure 1 Method overview. We combined two cohorts of CFW mice for 23 shared phenotypes. We identified 17 phenotypes with heritability significantly $>0$. Fifteen of these phenotypes had genetic correlations $>0.70$. We performed GWAS meta-analysis using these 15 phenotypes and obtained 70 genome-wide significant QTLs. 


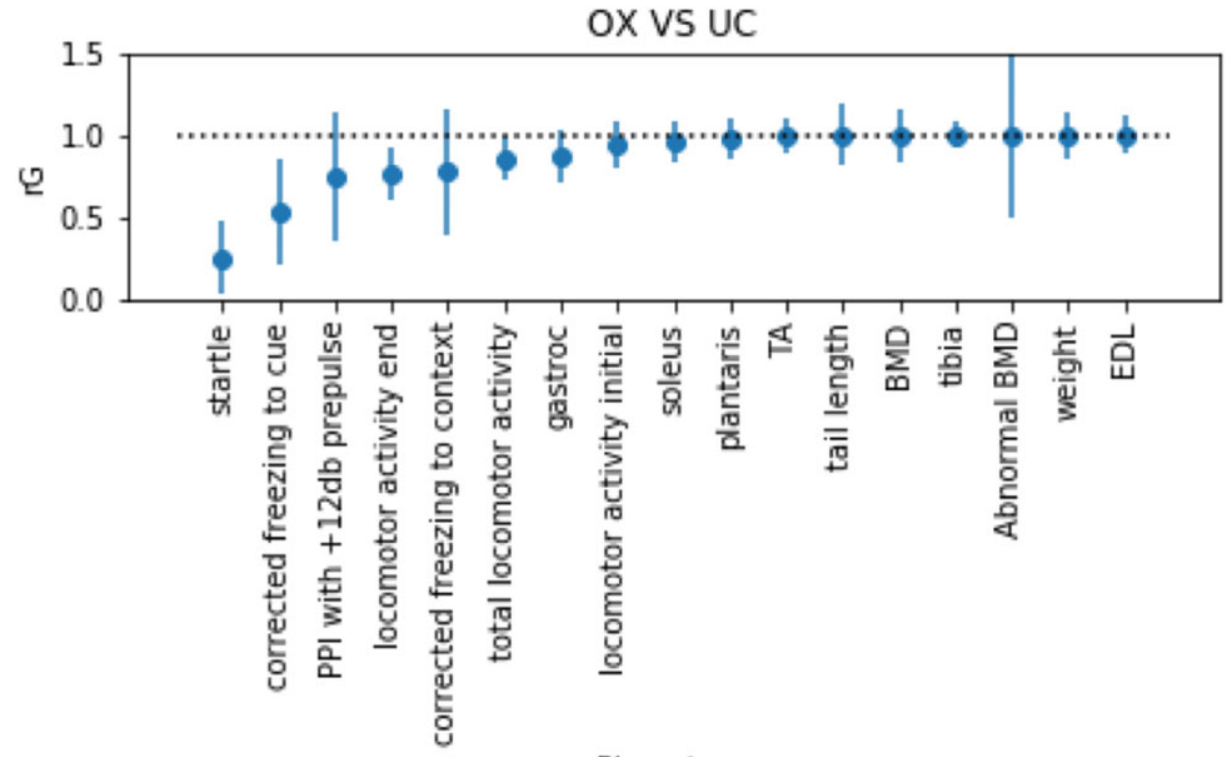

Phenotype

Figure 2 The genetic correlation between each pair of phenotypes mapped in OX and UC studies. Each dot represents the estimated genetic correlation, and the error bars show the $95 \%$ confidence intervals obtained using the standard errors. Only phenotypes with heritability significantly different from zero are shown. Phenotype names are defined in Supplementary Table S1.

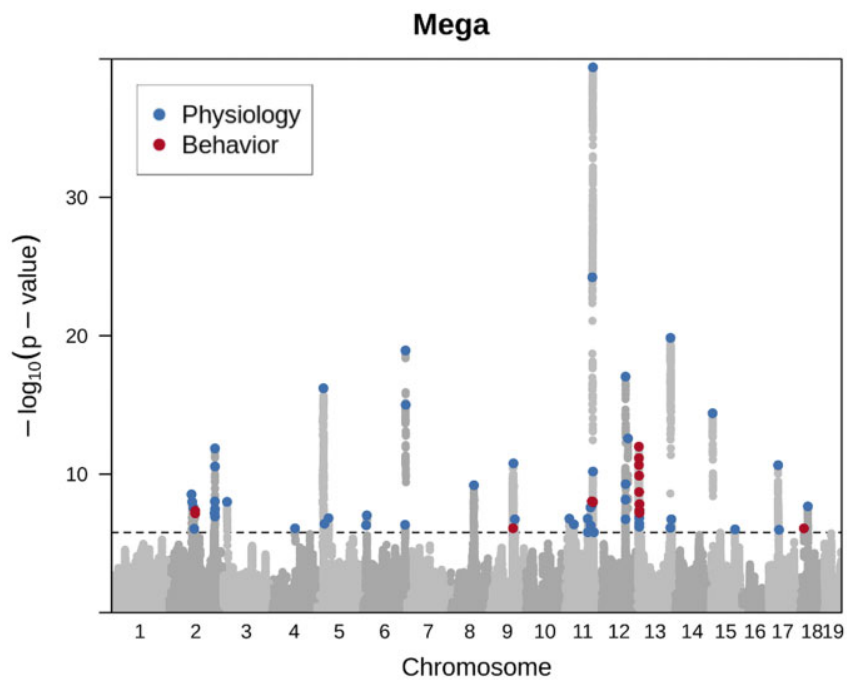

Figure 3 Porcupine plot showing a genome-wide representation of all QTLs identified for all traits. Light gray dots show association for the measures where a QTL was detected. SNPs at each locus that exceed a permutation derived significance threshold are marked with a colored dot, where blue represents physiological and red behavioral traits. Significance thresholds were derived using a decorrelated permutation approach, described in the methods section. While some of these QTLs represent closely linked loci for similar traits, some loci are shared between physiological (blue) and behavioral (red) traits.

Table S2) than the physiological traits. However, this may be in part due to differences in methodology between the OX and UC studies (see Methods).

\section{Mega-analysis}

We performed a mega-analysis of 23 traits in the combined OX and UC cohorts (3076 mice, Supplementary Data S3). We compared two different permutation-based methods ("naive" and "decorrelated") to obtain thresholds at a significance level of 0.05 (see Methods; Supplementary Figure S6). We used the more conservative decorrelated thresholds for our analysis. We identified 70 independent loci that were significant at an empirically derived - $\log$ P threshold of 5.79 (Figure 3).

The genetic architecture of the phenotypes is polygenic; namely many loci each contribute a small effect to the heritability (Supplementary Table S3). Figure 3 shows that the log P values for behavior (red dots in the figure) are on average less than those for physiological traits (blue dots). The absolute median effect size for the behavioral loci was 0.16 and 0.18 for physiological traits, indicating that the behavioral QTLs tended to have smaller effect sizes than the physiological QTLs. Supplementary Table S3 lists the positions of all genome-wide significant loci for every phenotype, giving their $\log \mathrm{P}$ and effect sizes.

\section{Confidence intervals}

We estimated the 95\% CI for every QTL using a previously published simulation procedure in which, for each locus, we randomly implanted causal SNPs that matched the true QTL's observed effect size and simulated phenotypes for all samples (Nicod et al. 2016). A local scan of the region using the mixed model was then performed using the simulated phenotypes, and the location and P-value of the top SNP was recorded. These simulations were used to compute the empirical distribution of the change in P-value between the most highly associated SNP and the causal SNP $(\Delta)$. The $95 \%$ CI was estimated as the maximum $\Delta$ among the lowest $95 \%$ of simulations. Supplementary Table S3 contains the CIs estimated for all QTLs found in the megaanalysis. Figure 4 shows several examples of CIs computed for two QTLs. Most CIs coincide with strong LD blocks, as expected (Figure 4A). However, in some cases, the CIs included variants with low correlation to the lead SNP (Figure 4B). Thus, the CIs are able to identify regions responsible for the peak signal in a less arbitrary way than simple LD thresholds. These CIs were computed using the LD pruned variants. We use these variants to simplify the simulations for the CI estimation and the subsequent finemapping. However, we add variants in LD for downstream analyses of the variants. Supplementary Figure S7 shows the 
A

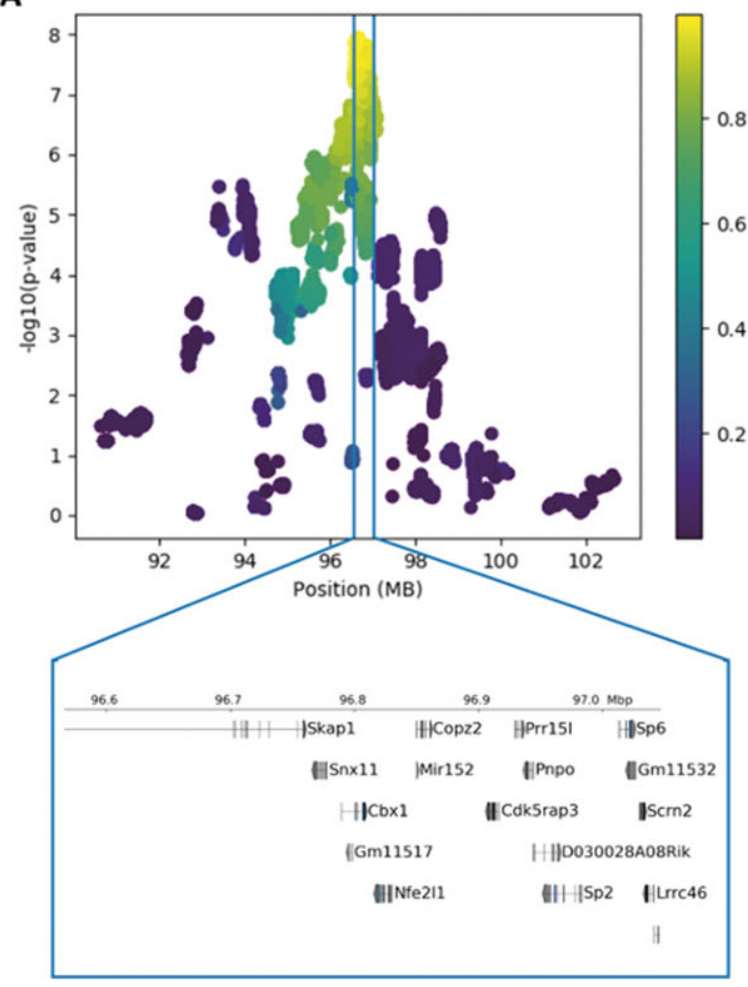

B

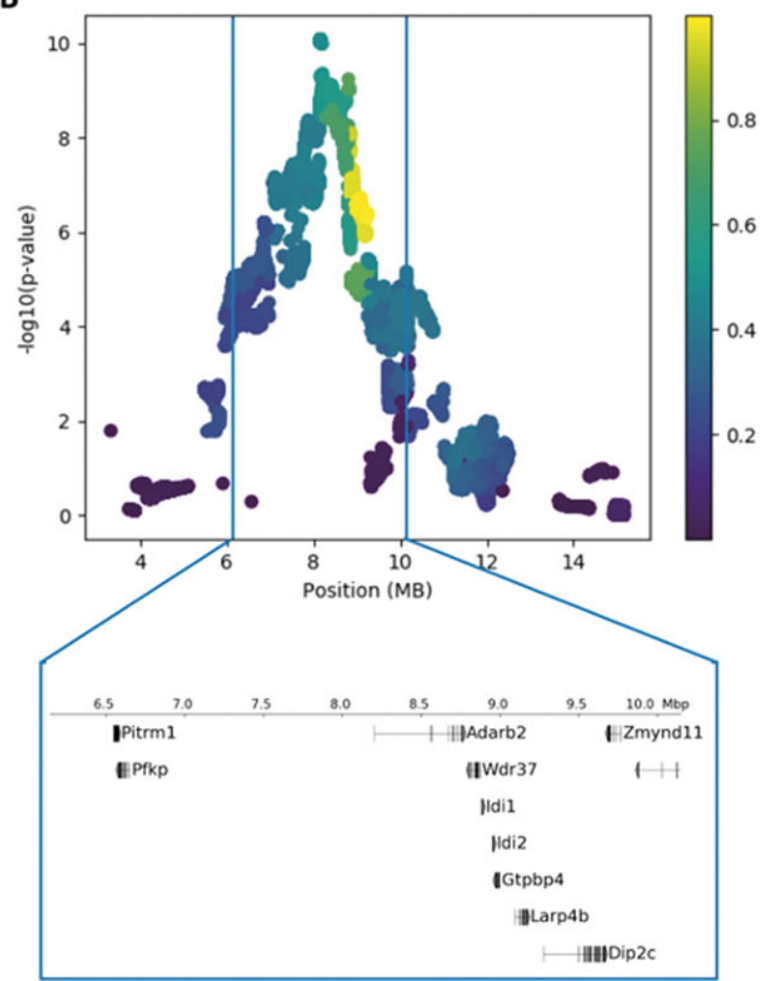

Figure 4 High-resolution mapping of two QTLs. Each point is a variant in the locus, and the color of the point corresponds to the correlation of that variant with the lead SNP. The vertical lines correspond to the $95 \%$ confidence interval estimated for the QTL. Genes falling within the confidence intervals are shown below the plot. (A) QTL for locomotor activity end (chr11:96653605). The confidence intervals fall along a block of variants in strong LD. (B) QTL for initial locomotor activity (chr13:9154368). The confidence intervals include many variants with low correlation to the lead SNP.

Table 1 Examples of fine-mapping QTLS

\begin{tabular}{lrrrrc}
\hline Phenotype & Chr & Position & All SNPs & Fm SNPs & Reduction \\
\hline Tibia & 6 & 145481081 & 15,815 & 169 & 0.99 \\
TA & 2 & 154645131 & 3,284 & 72 & 0.98 \\
Total locomotor & 2 & 89914086 & 1,366 & 43 & 0.97 \\
$\quad$ activity & 2 & 155505679 & 2,812 & 140 & 0.95 \\
Soleus & 11 & 100053320 & 20,037 & 2,980 & 0.85 \\
BMD &
\end{tabular}

"All SNPs" refers to the total number of SNPs used as input to the SusieR finemapping method, and "Fm SNPs" corresponds to the number of variants in the credible sets. "Reduction" is the percentage of decrease relative to the total number of SNPs used as input to the fine mapping.

distribution of the $95 \%$ CI widths of all QTLs, which range from 0.09-7.96 Mb, with a median of $1.3 \mathrm{Mb}$.

\section{Fine-mapping of mega-analysis loci}

In order to identify putative causal variants within the $95 \%$ CIs previously computed, we applied a fine-mapping framework called susieR (Wang et al. 2020). We used summary statistics from an LD pruned set $\left(R^{2}<0.99\right)$ of variants and the LD between these variants as input to susieR. SusieR uses these variants to compute a number of credible sets that are designed to contain the causal variant with high probability (i.e., 95\%). We obtained credible sets of SNPs for 62/70 QTLs for which the fine-mapping algorithm converged. Table 1 provides examples in which the fine-mapping was particularly effective. For example, a QTL we identified for tibia length (chr6:145481081) had over 15,000 SNPs in the $95 \%$ CI of the QTL, and after fine-mapping, the number of putative SNPs was only 169 (representing a 99\% reduction in the number of putative SNPs). The median number of variants in the credible sets was 932, and the median reduction in set size was 51\% (Supplementary Figure S8 and Data S4). Across all phenotypes and loci, we identified 94,177 variants in the credible sets or in high LD $\left(R^{2}>0.99\right)$ of variants in the credible sets. We used the variants within these credible sets as a high confidence set of variants for downstream analysis.

We generated gene-based annotations for the variants in the credible sets using the ANNOVAR software. Ninety-nine percentage of variants were in noncoding regions of the genome. 32 variants in the credible sets were nonsynonymous mutations within 22 coding genes (compared to 4 nonsynonymous variants in UC and 16 nonsynonymous variants in OX). We interrogated the NHGRI-EBI catalog of human GWAS and the Mouse Genome Informatics database to determine if any of these 22 genes have been previously associated with relevant traits of interest (Supplementary Table S4). Of the 22 coding genes identified, 11 were previously implicated in either human GWAS or KO mouse studies of similar traits (Supplementary Table S4).

\section{Colocalized traits}

We examined the effect of the 70 QTL significant in the megaanalysis on the other 23 traits, to determine the extent of colocalization between traits. We found that 45/70 QTLs had effects on more than one trait, after applying a Bonferroni correction $(P<5.1 e-05)$ for multiple testing of combinations of QTLs and other phenotypes. Phenotypes often derive from the same test (such as activity measured over different lengths of time or measures obtained from different muscles). To report our results we created two phenotype categories (Behavior and Physiology). Results are shown in Supplementary Figure S9 and Table S5. While the majority of these colocalized traits are between similar 
traits, such as locomotor activity at different time points, 20 of 45 QTLs had effects on at least one behavioral trait and one physiological trait. For example, a QTL for initial locomotor activity (chr11:96964818) was also associated with total locomotor activity, locomotor activity end, weight of TA, tibia length, BMD, and abnormal BMD. Similarly, a QTL for weight (chr13:9217096) was also associated with soleus, weight of TA, EDL, total locomotor activity, locomotor activity end, and locomotor activity initial.

\section{Replication}

Despite the high genetic correlations between phenotypes in the OX and UC studies (Figure 2), we observed a high rate of nonreplication of QTLs, where replication was defined as a genome-wide significant association in the "discovery" data set (OX or UC) and a P-value of 0.05 or lower in the other ("replication") data set. After mapping all traits in either just the OX or just the UC sample and applying empirically derived thresholds (-log P 5.68 for OX, 5.43 for UC), we identified 32 QTLs in the OX data set and 22 in the UC data set. Six of the 32 QTL discovered in OX replicated in the UC (19\%); of the 22 QTL identified in UC, 7 replicated in OX (32\%). There were also instances where the mega-analysis failed to confirm findings in a component study (12 of 22 loci from UC and 4 of 32 loci from OX, Supplementary Figure S10). Forty-one of 70 loci were significant in the mega-analysis but not significant in the component studies (Table 2).

We explored the contributions of power and confounders (which are presumed to reflect experimental differences between studies) to replication rates in our samples. When power is low, variants that pass the significance threshold are more likely to have inflated effect size estimates, meaning they are less likely to be replicated, a phenomenon that is often called "The Winner's Curse." Put another way, when power is low, the positive predictive value falls, so that for a given $\mathrm{P}$-value there will be more false positives.

We applied a statistical framework that jointly models Winner's Curse and study-specific heterogeneity due to confounding (Zou et al. 2020). The inputs to this method are the $z$-scores of the significant variants from the discovery study and the corresponding $z$-scores from the replication study. The method compares two random effects models: one that accounts for Winner's Curse (WC) and another that accounts for both Winner's Curse and study-specific heterogeneity due to confounding $(\mathrm{WC}+\mathrm{C})$. We compared the expected replication rates

Table 2 The number of QTLs significant in the mega-analysis ("mega-analysis") and the number of the QTLs from the megaanalysis that were also found in each of the component studies ("OX" and "UC")

\begin{tabular}{lccc}
\hline Phenotype & Mega-analysis & OX & UC \\
\hline Plantaris & 4 & 1 & 0 \\
Weight & 3 & 1 & 0 \\
Abnormal BMD & 2 & 2 & 1 \\
Startle habituation ratio & 1 & 0 & 0 \\
BMD & 8 & 4 & 3 \\
gastroc & 4 & 1 & 0 \\
Soleus & 6 & 3 & 1 \\
Total locomotor activity & 7 & 2 & 0 \\
EDL & 7 & 2 & 0 \\
Tibia & 10 & 8 & 3 \\
Locomotor activity initial & 7 & 1 & 0 \\
Tail length & 1 & 0 & 0 \\
TA & 7 & 3 & 0 \\
Locomotor activity end & 3 & 1 & 0 \\
\hline
\end{tabular}

Phenotype names are defined in Supplementary Table S1. under each of these random effects models to the observed replication rates. If the observed replication rate is well explained by the WC model, there is relatively little heterogeneity. However, if the observed replication rate is better explained by the $\mathrm{WC}+\mathrm{C}$ model, there may be heterogeneity between the studies.

We used the OX cohort as the discovery data set and UC cohort as the replication data set. We jointly modeled Winner's Curse and study-specific heterogeneity in nine phenotypes which had at least three significant loci [model parameters are not robustly estimated with fewer significant loci (Zou et al. 2020)]. We computed the expected replication rate under each model for each phenotype and compared these estimates with the observed replication between the two data sets. In order to estimate CIs we applied this method to 100 randomly divided sets of the OX sample (heterogeneity should not exist when a single study is randomly split in half into two cohorts). To display the results, we
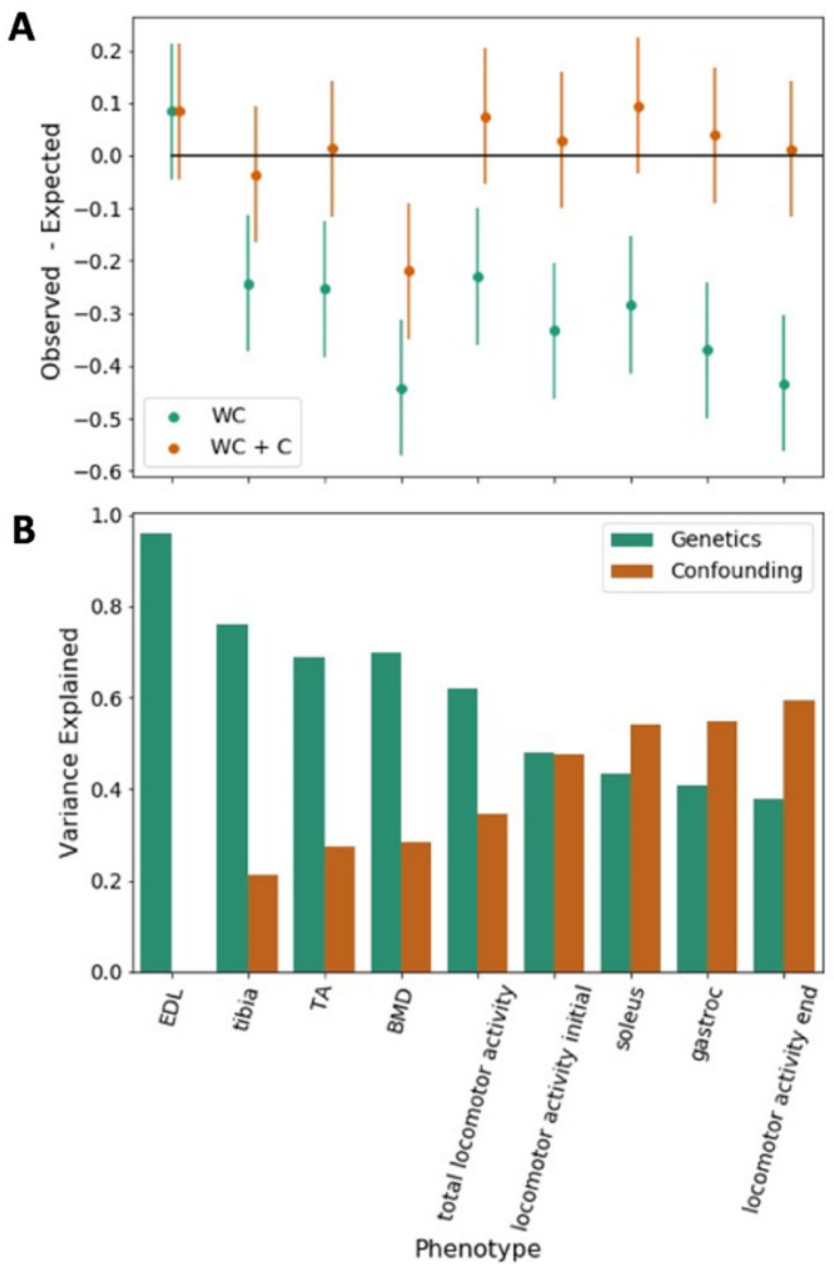

Figure 5 Replication analysis between OX and UC cohorts. (A) Difference between the observed and expected replication (y-axis) after accounting for Winner's Curse (green) and after accounting for both Winner's Curse and study-specific heterogeneity due to confounding (brown). Empirically computed $95 \%$ confidence intervals are shown as vertical bars. (B) Variance attributable to study-specific heterogeneity and genetics. The phenotypes are sorted by the amount of variance explained by genetics. There are six phenotypes with at least as much variance explained by genetics as study-specific heterogeneity (EDL, tibia, BMD, total locomotor activity, TA, and locomotor activity initial) and three phenotypes where the heterogeneity is more substantial (soleus, gastroc, and locomotor activity end). Phenotype names are defined in Supplementary Table S1. 
plotted the difference between the observed replication and the expected replication after correcting for Winner's Curse, which should be close to zero when the model explains the observed data.

Figure 5A shows the results modeling Winner's Curse (WC). The estimate for one phenotype, EDL, overlaps with zero, but in all other cases the Winner's Curse model predicts higher rates of replication than we observed. The WC $+\mathrm{C}$ model jointly models Winner's Curse and study-specific heterogeneity. Taking into account confounds in addition to Winner's Curse $(\mathrm{WC}+\mathrm{C})$ does a better job explaining the observed replication, as shown in Figure 5A. Only one phenotype, bone mineral density, deviates significantly from zero (Figure $5 \mathrm{~A}$ ). In this phenotype, replication was not explained well by either model.

We estimated the relative contribution of WC and studyspecific heterogeneity for each phenotype. Figure 5B shows that the relative contribution varies between phenotypes. Replication rate differences for EDLs depend almost entirely on WC while for soleus, gastroc, and locomotor activity end, confounds outweigh the contribution of WC. In general, behavioral phenotypes tended to have more heterogeneity than physiological phenotypes.

We returned to the results of the mega-analysis to categorize the robustness of the findings, based on the results of our replication analysis. For each of the genome-wide significant QTLs in the OX, UC, and mega-analysis studies, we corrected the observed effect sizes for WC using Zhong and Prentice (2008). This method takes into account the significance threshold used to detect the QTLs. We estimated the power using this corrected effect size estimate (Supplementary Table S6). As expected, power was on average higher in the mega-analysis than the component studies (Supplementary Figure S11). We then used these power estimates in combination with replication data and estimates of study-specific heterogeneity to divide the mega-analysis results into four categories (Table 3).

The first category consisted of a high confidence set of variants that were significant in the combined study and both component study. Eight of the 70 variants in four phenotypes were in this first category of variants. The second category consisted of variants that were significant in the combined study and at least one component study. Twenty-one of 70 variants in 12 phenotypes were in this second category of variants. The third category consisted of variants found in phenotypes without evidence of heterogeneity (excluding phenotypes without enough significant variants to determine the heterogeneity level). Fourteen of the 70 variants in five phenotypes fell in this category. Finally, the fourth category contained the remaining variants. Twenty-seven of the 70 variants in 12 phenotypes fell within this category. A summary of the QTLs found in each of the categories is shown in Supplementary Table S7.

\section{Discussion}

By combining phenotypes and genotypes from two independent laboratories, we identified 70 loci for 23 complex traits in a population of 3076 commercially available outbred mice. The large sample delivered a median QTL interval size of $1.1 \mathrm{Mb}$. Finemapping reduced the number of likely causal variants, with a median reduction in set size of 51\%. For all traits analyzed our results are consistent with a polygenic architecture, in which the vast majority of causal variants likely lie in noncoding parts of the genome, and with the existence of a considerable degree of pleiotropy, a pattern commonly recognized from GWAS of human complex traits (Visscher et al. 2017). As well as indicating the genetic architecture of the traits, our findings cast some light on the biology of the phenotypes we have mapped, allowed us to examine factors contributing to replication, and hence to assess the robustness of our findings. We discuss these points below.

To assess the robustness of our findings in the mega-analysis, we categorized QTLs by integrating replication data, heterogeneity estimates for each phenotype, and estimates of power in the mega-analysis. We use replication of findings in the OX and UC cohorts to determine the two highest confidence categories. Confidence category 1 consisted of QTLs that replicated in both cohorts. One example QTL in this category was associated with tibia length (chr12:83514944). Through fine-mapping and annotation of variants in the causal set, we implicated the Zfyve1 gene. This gene was also implicated by two other loci associated with gastroc (chr12:83517483) and TA (chr12:83514944) and is expressed highly in these tissues (Lionikas et al. 2012, 2013). Zfyve1 is known to bind to Ptdins3P (Lee et al. 2019), which is an important mediator of Akt/PKB kinase activation and upregulation of protein synthesis (Hemmings and Restuccia 2012), providing a potential mechanism for influencing variability in muscle weight. Zfyue1's link to Ptdins3P along with the GWAS associations with tibia length, gastroc weight and TA weight suggests that Zfyve1 may be involved in modification of the intracellular signal promoting growth.

Confidence category 2 contained QTLs that replicated in one component study but not the other. These associations are less likely to be driven by differences between studies or Winner's Curse, since they were also significant in a component study with fewer samples than the mega-analysis. For example, chr11:94758993 was associated with abnormal bone mineral density in the mega-analysis and was replicated in the OX study but not the UC study. Through our fine-mapping and nonsynonymous variant annotation, we implicated the gene Tmem92, which has been previously found to be associated with heel bone mineral density in humans in a number of studies (Kemp et al. 2017; Kim 2018; Kichaev et al. 2019; Morris et al. 2019). Additionally, the Tbx18 gene which was implicated by a confidence level 2 QTL for plantaris weight (chr9:87855594), was previously associated with body size phenotypes in human GWAS studies and mouse KO studies (Wu et al. 2013; Lotta et al. 2018; Kichaev et al. 2019; Pulit et al. 2019).

Confidence category 3 contains QTLs that did not replicate in component studies. However, these QTLs were found in phenotypes with relatively lower heterogeneity and had high estimated levels of power (>0.5) in the mega-analysis after correcting for Winner's Curse. A QTL for EDL weight (chr13:113025220) in confidence category 3 implicated gene Cdc20b, which has been previously associated with BMI adjusted waist circumference in human GWAS (Zhu et al. 2020). The estimated power level for this variant was $11 \%$ in the OX study, $14 \%$ in the UC study, and $67 \%$ in the mega-analysis. QTLs in confidence level 3 are likely to be true associations that were identified due to the increase in power in the mega-analysis.

In general, we observe that genes implicated using high confidence QTLs are more likely to have support in previously published results. For example, we identified seven olfactory receptor genes from two QTLs associated with EDL weight and body weight that were in the lowest category of confidence (4). Additionally, the EDL QTL (chr2:86662262) had low estimated power after correcting for Winner's Curse, and the body weight QTL (chr17:37094289) had a high estimated level of heterogeneity between studies. None of the seven genes have been previously associated with related phenotypes in the literature and are 


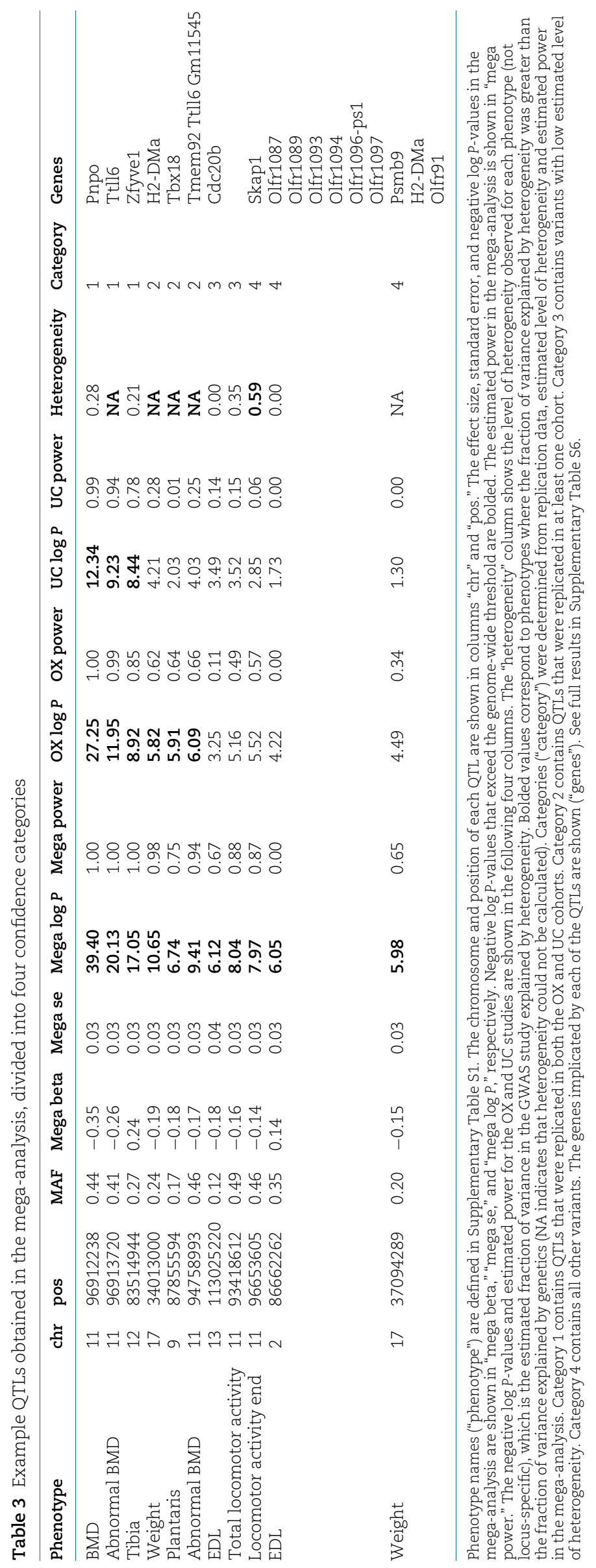


unlikely to be true associations. However, there is evidence to support the candidacy of genes at some of the QTLs in this category. For example, a QTL for soleus weight (chr13:9242435) implicates Larp $4 b$, which has been previously associated with atypical femoral fracture and ankle injury in human GWAS (Kim et al. 2017; Kharazmi et al. 2019). Since the soleus is the plantar flexor muscle of the ankle, it is likely to play a role in these two phenotypes. While we estimated substantial amounts of heterogeneity for the locomotor activity end phenotype and the soleus weight phenotype, previous findings may indicate that these genes may have been identified in spite of the heterogeneity we observed.

While these examples highlight genes that have previously been associated with similar phenotypes in mice or humans, other genes (Pnpo, Gm11545, Psmb9, and Ttll6) represent novel targets for functional validation in follow-up studies. In particular, Pnpo lies at the bone mineral density QTL chr11:96912238, in confidence category 1 . The bone mineral density phenotype had relatively low estimated levels of heterogeneity between the OX and UC cohorts, and the power of the mega-analysis after correcting for Winner's Curse was close to $100 \%$.

Our results also provided us with an opportunity to identify the factors that contribute to replication rates. When we investigated replication between component studies we found that about a fifth (19\%) of QTL discovered in OX replicated in UC and just less than one-third (32\%) of QTL identified in UC replicated in OX. We explored reasons for the low rates of replication and came to the following conclusions.

Unknown confounds between laboratories limited replication, but this depended on the phenotype. By applying a method that detects the effect of heterogeneity, we observed that for one phenotype there was no impact of heterogeneity (weight of the EDL muscle), while on others the impact was substantial (up to $60 \%$ of the variance explained in the case of weight of the plantaris muscle). Some of the differences likely reflect systematic differences between the way the mice were treated at the two testing sites: mice born in Portage, Michigan, were shipped either to Chicago by truck $(\sim 200 \mathrm{~km})$ or to Oxford by truck and plane $(\sim 6100 \mathrm{~km})$. Other factors may include differences in the way the animals were handled or a variety of other laboratory specific differences.

Current human GWAS usually report results only from their meta-analyses, following the argument that joint analysis is more powerful than replication-based analyses (Skol et al. 2006), and rarely rely on replication as a touchstone for the dependability of their findings. Results obtained in this way are usually regarded as robust (Marigorta et al. 2018). However, replication rates may be inconsistent with the $P$-values and effect sizes reported in discovery cohorts; for example, a replication rate of $48 \%$ was found in a survey of 100 papers (Palmer and Pe'er 2017).

The bulk of human GWAS findings since 2008 are replications of associations that have been described at least twice (Marigorta et al. 2018), thus allaying concerns that the findings are not robust. The same cannot be said for mouse GWAS, where it is rare for a trait to be mapped multiple times at all, much less to be mapped multiple times in the same population. Furthermore, the genetic structure of mouse mapping populations is quite unlike that of outbred human populations. For example, no human population consists of fully inbred individuals, a commonly used design in mouse experiments [as in the recombinant inbred strains of the collaborative cross, or the inbred strains that constitute the hybrid mouse diversity panel (Flint and Eskin 2012)]. Replication across such different populations is more difficult than within a population [as already documented for human studies (Palmer and Pe'er 2017)]. We show here that obtaining robust results in mice is demanding because of difficulties in obtaining a replication population, the impact of Winner's curse and phenotype specific confounds. As a first step we derive categories of confidence to our mapping results by combining information about replication, study-specific heterogeneity, and power. We thus categorized loci into one of four confidence levels. Our approach helps alleviate concerns about reproducibility (Button et al. 2013) and can be used to prioritize QTLs for follow-up studies, but needs further development to produce a systematic method to evaluate confidence quantitatively, rather than categorically as we have attempted here. This framework may also be applicable to human GWAS especially when replicating in admixed populations, when phenotyping may be difficult.

\section{Data availability}

The authors affirm that all data necessary for confirming the conclusions of the article are present within the article, figures, and tables. The data and results presented in this paper require no additional permissions for use. Raw data from our study is deposited in Dryad: https://datadryad.org/stash/share/P3DJwz6Ligfti43XmQcQ AWzLQaN7a3Rt8S7y5KMx90g (Last accessed November 18, 2021). Code used to perform the analyses in this paper can be found at https://github.com/jzou1115/CFW_code.

Supplementary material is available at G3 online.

\section{Funding}

This work was partially supported by National Institutes of Health grants [R01MH115979 (J.F.), R01GM097737 and P50DA037844 (A.A.P)]. J.Z. is supported by a National Science Foundation Graduate Research Fellowship under Grant DGE1650604. Publication charges for this article have been funded by 1R01MH115979.

J.F., A.A.P., and R.M. conceived the study. J.Z., J.F., and S.G. performed the bioinformatics analysis. C.P. and J.N. prepared the phenotypes. R.W.D. generated the genotypes. J.Z., C.P., S.G, N.C, A.L. A.A.P., and J.F. wrote the manuscript. All authors read and approved the final manuscript.

\section{Conflicts of interest}

The authors declare that no conflict of interest.

\section{Literature cited}

Abney M. 2015. Permutation testing in the presence of polygenic variation. Genet Epidemiol. 39:249-258.

Bassett JH, van der Spek A, Gogakos A, Williams GR. 2012. Quantitative X-ray imaging of rodent bone by Faxitron. Methods Mol Biol. 816:499-506.

Button KS, Ioannidis JP, Mokrysz C, Nosek BA, Flint J, et al. 2013. Power failure: why small sample size undermines the reliability of neuroscience. Nat Rev Neurosci. 14:365-376.

Cheng R, Palmer AA. 2013. A simulation study of permutation, bootstrap, and gene dropping for assessing statistical significance in the case of unequal relatedness. Genetics. 193:1015-1018.

Chesler EJ. 2014. Out of the bottleneck: the Diversity Outcross and Collaborative Cross mouse populations in behavioral genetics research. Mamm Genome. 25:3-11. 
Chitre AS, Polesskaya O, Holl K, Gao J, Cheng R, et al. 2020. Genome-Wide Association Study in 3,173 outbred rats identifies multiple loci for body weight, adiposity, and fasting glucose. Obesity (Silver Spring). 28:1964-1973.

Collaborative Cross Consortium. 2012. The genome architecture of the collaborative cross mouse genetic reference population. Genetics. 190:389-401.

Crabbe JC, Wahlsten D, Dudek BC. 1999. Genetics of mouse behavior: interactions with laboratory environment. Science. 284: 1670-1672.

Davies RW, Flint J, Myers S, Mott R. 2016. Rapid genotype imputation from sequence without reference panels. Nat Genet. 48:965-969.

Flint J, Eskin E. 2012. Genome-wide association studies in mice. Nat Rev Genet. 13:807-817.

Ghazalpour A, Rau CD, Farber CR, Bennett BJ, Orozco LD, et al. 2012. Hybrid mouse diversity panel: a panel of inbred mouse strains suitable for analysis of complex genetic traits. Mamm Genome. 23:680-692.

Hemmings BA, Restuccia DF. 2012. PI3K-PKB/Akt pathway. Cold Spring Harb Perspect Biol. 4:a011189.

Kang EY, Han B, Furlotte N, Joo JW, Shih D, et al. 2014. Meta-analysis identifies gene-by-environment interactions as demonstrated in a study of 4,965 mice. PLoS Genet. 10:e1004022.

Kemp JP, Morris JA, Medina-Gomez C, Forgetta V, Warrington NM, et al. 2017. Identification of 153 new loci associated with heel bone mineral density and functional involvement of GPC6 in osteoporosis. Nat Genet. 49:1468-1475.

Kharazmi M, Michaelsson K, Schilcher J, Eriksson N, Melhus H, et al. 2019. A genome-wide association study of bisphosphonate-associated atypical femoral fracture. Calcif Tissue Int. 105:51-67.

Kichaev G, Bhatia G, Loh PR, Gazal S, Burch K, et al. 2019. Leveraging polygenic functional enrichment to improve GWAS power. Am J Hum Genet. 104:65-75.

Kim SK. 2018. Identification of 613 new loci associated with heel bone mineral density and a polygenic risk score for bone mineral density, osteoporosis and fracture. PLoS One. 13:e0200785.

Kim SK, Kleimeyer JP, Ahmed MA, Avins AL, Fredericson M, et al. 2017. Two genetic loci associated with ankle injury. PLoS One. 12: e0185355.

Lee JW, Nam H, Kim LE, Jeon Y, Min H, et al. 2019. TLR4 (toll-like receptor 4) activation suppresses autophagy through inhibition of FOXO3 and impairs phagocytic capacity of microglia. Autophagy. 15:753-770.

Lionikas A, Kilikevicius A, Bunger L, Meharg C, Carroll AM, et al. 2013. Genetic and genomic analyses of musculoskeletal differences between BEH and BEL strains. Physiol Genomics. 45:940-947.

Lionikas A, Meharg C, Derry JM, Ratkevicius A, Carroll AM, Vandenbergh DJ, et al. 2012. Resolving candidate genes of mouse skeletal muscle QTL via RNA-Seq and expression network analyses. BMC Genomics. 13:592.

Lotta LA, Wittemans LBL, Zuber V, Stewart ID, Sharp SJ, et al. 2018. Association of genetic variants related to gluteofemoral vs abdominal fat distribution with type 2 diabetes, coronary disease, and cardiovascular risk factors. JAMA. 320:2553-2563.

Marigorta UM, Rodriguez JA, Gibson G, Navarro A. 2018. Replicability and prediction: lessons and challenges from GWAS. Trends Genet. 34:504-517.

Morris JA, Kemp JP, Youlten SE, Laurent L, Logan JG, et al.; 23andMe Research Team. 2019. An atlas of genetic influences on osteoporosis in humans and mice. Nat Genet. 51:258-266.

Nicod J, Davies RW, Cai N, Hassett C, Goodstadt L, et al. 2016. Genome-wide association of multiple complex traits in outbred mice by ultra-low-coverage sequencing. Nat Genet. 48: 912-918.

Palmer AA, Airey DC. 2003. Inappropriate choice of the experimental unit leads to a dramatic overestimation of the significance of quantitative trait loci for prepulse inhibition and startle response in recombinant congenic mice. Neuropsychopharmacology. 28: 818; author reply 819

Palmer AA, Dulawa SC, Mottiwala AA, Conti LH, Geyer MA, et al. 2000. Prepulse startle deficit in the Brown Norway rat: a potential genetic model. Behav Neurosci. 114:374-388.

Palmer AA, Printz DJ, Butler PD, Dulawa SC, Printz MP. 2004. Prenatal protein deprivation in rats induces changes in prepulse inhibition and NMDA receptor binding. Brain Res. 996:193-201.

Palmer C, Pe'er I. 2017. Statistical correction of the Winner's Curse explains replication variability in quantitative trait genome-wide association studies. PLoS Genet. 13:e1006916.

Parker CC, Gopalakrishnan S, Carbonetto P, Gonzales NM, Leung E, et al. 2016. Genome-wide association study of behavioral, physiological and gene expression traits in outbred CFW mice. Nat Genet. 48:919-926.

Parker CC, Palmer AA. 2011. Dark matter: are mice the solution to missing heritability? Front Genet. 2:32.

Pe'er I, Yelensky R, Altshuler D, Daly MJ. 2008. Estimation of the multiple testing burden for genomewide association studies of nearly all common variants. Genet Epidemiol. 32:381-385.

Pulit SL, Stoneman C, Morris AP, Wood AR, Glastonbury CA, et al.; GIANT Consortium. 2019. Meta-analysis of genome-wide association studies for body fat distribution in 694649 individuals of European ancestry. Hum Mol Genet. 28:166-174.

Purcell S, Neale B, Todd-Brown K, Thomas L, Ferreira MA, et al. 2007. PLINK: a tool set for whole-genome association and population-based linkage analyses. Am J Hum Genet. 81:559-575.

R Development Core Team. 2010. R: A Language and Environment for Statistical Computing. Vienna, Austria: R Foundation for Statistical Computing.

Schmidt C, Gonzaludo NP, Strunk S, Dahm S, Schuchhardt J, et al. 2008. A meta-analysis of QTL for diabetes-related traits in rodents. Physiol Genomics. 34:42-53.

Shanahan NA, Holick Pierz KA, Masten VL, Waeber C, Ansorge M, et al. 2009. Chronic reductions in serotonin transporter function prevent 5-HT1B-induced behavioral effects in mice. Biol Psychiatry. 65:401-408.

Skol AD, Scott LJ, Abecasis GR, Boehnke M. 2006. Joint analysis is more efficient than replication-based analysis for two-stage genome-wide association studies. Nat Genet. 38:209-213.

Speed D, Hemani G, Johnson MR, Balding DJ. 2012. Improved heritability estimation from genome-wide SNPs. Am J Hum Genet. 91: 1011-1021.

Sun L, Dimitromanolakis A, Faye LL, Paterson AD, Waggott D, et al.; DCCT/EDIC Research Group. 2011. BR-squared: a practical solution to the winner's curse in genome-wide scans. Hum Genet. 129:545-552.

Valdar W, Solberg LC, Gauguier D, Cookson WO, Rawlins JN, et al. 2006. Genetic and environmental effects on complex traits in mice. Genetics. 174:959-984.

Visscher PM, Brown MA, McCarthy MI, Yang J. 2012. Five years of GWAS discovery. Am J Hum Genet. 90:7-24.

Visscher PM, Wray NR, Zhang Q, Sklar P, McCarthy MI, Brown MA, et al. 2017. 10 years of GWAS discovery: biology, function, and translation. Am J Hum Genet. 101:5-22.

Wang G, Sarkar A, Carbonetto P, Stephens M. 2020. A simple new approach to variable selection in regression, with application to genetic fine mapping. J R Stat Soc B. 82:1273-1300. 
Wang K, Li M, Hakonarson H. 2010. ANNOVAR: functional annotation of genetic variants from high-throughput sequencing data. Nucleic Acids Res. 38:e164.

Wu SP, Dong XR, Regan JN, Su C, Majesky MW. 2013. Tbx18 regulates development of the epicardium and coronary vessels. Dev Biol. 383:307-320.

Wuschke S, Dahm S, Schmidt C, Joost HG, Al-Hasani H. 2007. A meta-analysis of quantitative trait loci associated with body weight and adiposity in mice. Int J Obes (Lond). 31:829-841.

Xiao R, Boehnke M. 2009. Quantifying and correcting for the winner's curse in genetic association studies. Genet Epidemiol. 33: 453-462.

Yang J, Lee SH, Goddard ME, Visscher PM. 2011. GCTA: a tool for genome-wide complex trait analysis. Am J Hum Genet. 88:76-82.

Yee BK, Chang T, Pietropaolo S, Feldon J. 2005. The expression of prepulse inhibition of the acoustic startle reflex as a function of three pulse stimulus intensities, three prepulse stimulus intensities, and three levels of startle responsiveness in C57BL6/J mice. Behav Brain Res. 163:265-276.
Zhong H, Prentice RL. 2008. Bias-reduced estimators and confidence intervals for odds ratios in genome-wide association studies. Biostatistics. 9:621-634.

Zhou X, St Pierre CL, Gonzales NM, Zou J, Cheng R, et al. 2020. Genome-Wide Association Study in two cohorts from a multi-generational mouse advanced intercross line highlights the difficulty of replication due to study-specific heterogeneity. G3 (Bethesda). 10:951-965.

Zhou X, Stephens M. 2012. Genome-wide efficient mixed-model analysis for association studies. Nat Genet. 44:821-824.

Zhu Z, Guo Y, Shi H, Liu CL, Panganiban RA, et al. 2020. Shared genetic and experimental links between obesity-related traits and asthma subtypes in UK Biobank. J Allergy Clin Immunol. 145: 537-549.

Zou J, Zhou J, Faller S, Brown R, Eskin E. 2020. Accurate modeling of replication rates in genome-wide association studies by accounting for winner's curse and study-specific heterogeneity. bioRxiv. 856898 .

Communicating editor: T. Matise 